\title{
A NEW PROOF OF THE EQUTVALENCE OF THE HAHN-BANACH EXTENSION AND THE LEAST UPPER BOUND PROPERTIES
}

\author{
A. D. IOFFE
}

\begin{abstract}
The paper contains a new proof of the fact that the Hahn-Banach majorized extension theorem for linear operators is valid iff the range ordered space is conditionally complete. The proof is based on quite different principles than the original proof of Bonnice, Silverman and To. The key element is a reformulation of the extension problem in terms of linear selections of special convex-valued mappings called fans.
\end{abstract}

Let $Y$ be a real linear space ordered by a cone $K$ (which may be a wedge). Consider the following two properties:

Hahn-Banach extension property (HBEP). For any linear space $X$, any linear subspace $L \subset X$, any sublinear mapping $P: X \rightarrow Y$ and any linear operator $B$ : $L \rightarrow Y$ such that $B x \leqslant P(x)$ for all $x \in L$, there is a linear operator $A: X \rightarrow Y$ such that

$$
A x \leqslant P(x), \quad \forall x \in X, \text { and } A x=B x, \quad \forall x \in L ;
$$

Least upper bound property (LUBP). Any set $Q \subset Y$ bounded from above has a least upper bound; in other words, there is an element sup $Q \in Y$ (not necessarily unique) such that $y \leqslant \sup Q$ for any $y \in Q$ and $y<z$ for all $y \in Q$ implies $\sup Q \leqslant z$.

THEOREM A. An ordered space $Y$ has the Hahn-Banach extension property if and only if it has the least upper bound property.

This theorem has a long history which probably started (as far as more than one dimensional $Y$ is concerned) with Kantorovič's paper [6] where the implication LUBP $\Rightarrow$ HBEP was proved for $X$ being a normed space and $P$ an abstract norm in $X$. We refer to [3] for a proof of the implication in a general situation. Silverman and Yen [9] (see also [3]) proved that HBEP implies LUBP if $K$ is linearly closed (intersection of $K$ with any line is closed in the natural topology of the line). Finally, Bonnice and Silverman [1], [2] and To [10] proved that HBEP itself implies linear closedness of the positive cone and hence LUBP.

Received by the editors August 18, 1979 and, in revised form, December 10, 1979 and July 7, 1980. 1980 Mathematics Subject Classification. Primary 46A40, 47B55, 47D20.

Key words and phrases. Ordered vector space, sublinear operator, Hahn-Banach extension, convexvalued mapping, fan, binary intersection property, least upper bound, conditionally complete order. 
We offer here a new proof based on the utilization of a new class of objects called fans (which are homogeneous subadditive convex-valued mappings). Occasionally, they did appear earlier (see for instance [7] just in connection with the Hahn-Banach theorem, also Rockafellar considered similar objects in connection with monotone processes which, contrary to fans, are superadditive-the results however remained unpublished) but now it becomes clear that for certain purposes, say, in nonsmooth or convex analysis they are as natural as linear operators [4], [5].

Theorem A will be obtained here as an easy corollary of another (and perhaps more general) Theorem B. Compared to the proof given by Bonnice, Silverman and To, the proof of Theorem B seems to be simpler and more natural because it is purely algebraic (as well as is the statement), not connected with any ordered structure and not incorporating any geometrical argumentation.

Definition 1. Let $X$ and $Z$ be linear spaces. A set-valued mapping $\mathcal{Q}: X \rightarrow Z$ is called fan if

(i) the sets $Q(x)$ are nonempty and convex for any $x \in X$;

(ii) $0 \in \mathbb{Q}(0)$;

(iii) $\mathbb{Q}(\lambda x)=\lambda \mathscr{Q}(x), \forall \lambda>0, \forall x \in X$;

(iv) $\mathbb{Q}(x+u) \subset \mathbb{Q}(x)+\mathbb{Q}(u), \forall x, u \in X$.

A fan is called odd if $\mathscr{Q}(-x)=-\mathscr{Q}(x)$ so that (iii) holds for any $\lambda \neq 0$, not necessarily positive.

In this paper we deal only with odd fans.

Definition 2. A linear operator $A: X \rightarrow Z$ is called a selection of $\mathbb{Q}$ if $A x \in$ $\mathcal{Q}(x)$ for any $x \in X$. We shall say that $\mathbb{Q}$ has the linear extension property if any selection of $\mathbb{Q}$ defined on a subspace of $X$ can be extended to a selection of $\mathbb{Q}$ defined on all of $X$.

Definition 3. Let $\mathcal{C}$ be a family of subsets of $Z$. We shall say that $\mathcal{C}$ has the binary intersection property if any collection of elements of $e$ has nonempty intersection, provided each two of them meet each other.

THEOREM B. Let $Z$ be a linear space, and let $\mathcal{C}$ be a family of convex subsets of $Z$ which is closed under translations, summation and multiplication by scalars. Then the following two statements are equivalent:

(a) $\mathcal{C}$ has the binary intersection property;

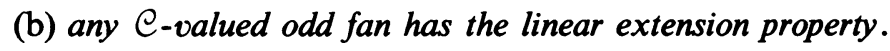

REMARK. Implication (a) $\Rightarrow$ (b) was actually proved in [7]. For this implication to be valid, it is sufficient to assume that $\mathcal{C}$ is only translation invariant.

First we shall show that Theorem A does follow from Theorem B. If $y, v \in Y$, $y<v$, then $[y, v]=\{q \in Y \mid y \leqslant q \leqslant v\}$ is the corresponding bounded order interval. It is said that $Y$ has the Riesz decomposition property (RDP) if

$$
[y, v]+\left[y^{\prime}, v^{\prime}\right]=\left[y+y^{\prime}, v+v^{\prime}\right]
$$

for any pair of order intervals $[y, v],\left[y^{\prime}, v^{\prime}\right]$.

LEMMA 1. If $Y$ has either LUBP or HBEP, then $Y$ has RDP.

Proof. Implication LUBP $\Rightarrow R D P$ is elementary and well known (cf. [8]). 
Assume HBEP. Fix two order intervals $[y, v]$ and $\left[y^{\prime}, v^{\prime}\right]$ and consider the following mapping $P: R^{2} \rightarrow Y$ :

$$
P(t, s)=(1 / 2)\left(|t|(v-y)+t(v+y)+|s|\left(v^{\prime}-y^{\prime}\right)+s\left(v^{\prime}+y^{\prime}\right)\right) .
$$

Since $y<v$ and $y^{\prime} \leqslant v^{\prime}$, this mapping is sublinear. We have $P(1,1)=v+v^{\prime}$, $P(-1,-1)=-y-y^{\prime}$ so that $[-P(-1,-1), P(1,1)]=\left[y+y^{\prime}, v+v^{\prime}\right]$. Let $q \in$ $\left[y+y^{\prime}, v+v^{\prime}\right]$. Then $\lambda q \leqslant P(\lambda, \lambda)$ for all $\lambda$; hence in view of HBEP, there is $A$ : $R^{2} \rightarrow Y$ such that $A(t, s) \leqslant P(t, s)$ for all $t, s$ and $A(1,1)=q$. Denote $q^{\prime}=A(1,0)$, $q^{\prime \prime}=A(0,1)$. Then

$$
\begin{aligned}
q^{\prime} & \leqslant P(1,0)=v, & -q^{\prime}=A(-1,0) \leqslant P(-1,0)=-y ; \\
q^{\prime \prime} & \leqslant P(0,1)=v^{\prime}, & -q^{\prime \prime}=A(0,-1) \leqslant P(0,-1)=-y^{\prime} ;
\end{aligned}
$$

so that $q^{\prime} \in[y, v], q^{\prime \prime} \in\left[y^{\prime}, v^{\prime}\right], q=q^{\prime}+q^{\prime \prime}$ which implies that $\left[y+v, y^{\prime}+v^{\prime}\right] \subset$ $[y, v]+\left[y^{\prime}, v^{\prime}\right]$. The converse inclusion is trivial.

LEMma 2. Assume that $Y$ has RDP. Then the following two properties are equivalent:

(a) $\mathcal{Q}$ is an odd fan from $X$ into $Y$ whose values are bounded order intervals;

(b) $\mathbb{Q}(x)=[-P(x), P(x)]$, where $P: X \rightarrow Y$ is sublinear.

Proof. Implication (b) $\Rightarrow$ (a) can be verified by direct calculation; in particular, subadditivity of $Q$ follows from RDP. Now let (a) hold. Denote $L=K \cap(-K)$, and let $L^{\prime}$ be a complementary space. If $I$ is a bounded order interval in $Y$, then there are unique $p, q \in L^{\prime}$ such that $I=[p, q]$. Therefore $\mathcal{Q}(x)=[P(x), Q(x)]$, where $Q, P: X \rightarrow L^{\prime}$. It follows immediately from the properties of fans that $Q$ is sublinear and $P(x)=-Q(-$.$) (due to oddness).$

LEMMA 3. If $Y$ has RDP, then the collection of bounded order intervals in $Y$ has the binary intersection property iff $Y$ has $L U B P$.

Proof. See [3].

LEMMA 4. If $Y$ has RDP, then $Y$ has HBEP iff any odd fan whose values are bounded order intervals in $Y$ has the linear extension property.

Proof. This is obvious from Lemma 2.

Theorem A follows immediately from Theorem B and these lemmas. (Observe that in view of Lemma 1, the collection of bounded order intervals in $Y$ is closed under summation, provided either LUBP or HBEP is valid. It is obvious that this collection of convex sets is always closed under translations and multiplication by scalars.)

Now we proceed with the proof of Theorem B.

Lemma 5. Let $\mathbb{Q}$ be an odd fan from $X$ into $Z$, and let $B$ be a linear selection of $\mathbb{Q}$ defined on a subspace $L \subset X$ of codimension one. Then $B$ can be extended to a linear selection of $\mathbb{Q}$ defined on all of $X$ if and only if

$$
\bigcap_{x \in L}(\mathscr{Q}(x+u)-B x) \neq \varnothing
$$

for any $u \notin L$. 
Proof. If (1) holds and $z$ belongs to the intersection, then we set $A(x+t u)=$ $B x+t z$ so that (when $t \neq 0$ )

$$
\mathbb{Q}(x+t u)-B x=t(\mathbb{Q}((x / t)+u)-B(x / t)) \ni t z
$$

which shows that $A(x+t u) \in \mathbb{Q}(x+t u)$.

Conversely, if an extension $A$ exists, then setting $z=A u$, we have $A(x+u)=$ $B x+z \in \mathbb{Q}(x+u)$ so that $z \in \mathbb{Q}(x+u)-B x$ for all $x \in L$.

Proof of Theorem B. (a) $\Rightarrow$ (b). The proof of this part is standard for theorems of Hahn-Banach type. The only thing we need to prove is the following: if $\mathbb{Q}$ is a fan from $X$ into $Z$ whose values belong to $\mathcal{C}$, if $B$ is a selection of $\mathcal{Q}$ defined on a subspace $L \subset X$ and if $M$ is a subspace of $X$ such that $L \subset M$ and $\operatorname{dim} M / L=1$, then there is a selection of $\mathcal{Q}$ defined on $M$ which coincides with $B$ on $L$. The rest is obtained by standard induction arguments.

For this purpose, we need to verify (in view of Lemma 5) that

$$
\left(\mathscr{Q}\left(x_{1}+u\right)-B x_{1}\right) \cap\left(\mathbb{Q}\left(x_{2}+u\right)-B x_{2}\right) \neq \varnothing \quad\left(x_{1}, x_{2} \in L\right)
$$

and then apply Lemma 5 having in mind that $\mathcal{C}$ has the binary intersection property. The simple calculation $0 \in \mathbb{Q}\left(x_{1}-x_{2}\right)-B\left(x_{1}-x_{2}\right) \subset \mathbb{Q}\left(x_{1}+u\right)-$ $\mathbb{Q}\left(x_{2}+u\right)-B\left(x_{1}-x_{2}\right)=\left(\mathbb{Q}\left(x_{1}+u\right)-B x_{1}\right)-\left(\mathbb{Q}\left(x_{2}+u\right)-B x_{2}\right)$ completes the proof.

(b) $\Rightarrow$ (a). Let $\left\{C_{i}\right\}_{i \in I}$ be a family of elements of $\mathcal{C}$ such that any two sets of the family meet each other. Let $X$ be the direct sum of $I$ copies of $R$, in other words, $X$ is the space of all real-valued functions on $I$ which assume nonzero values only in finitely many points. Then the set-valued mapping $Q(x)=\sum_{i \in I} x(i) C_{i}$ from $X$ into $Z$ is well defined. It is easy to see that $Q$ is a fan. Indeed, properties (i)-(iii) are obvious as well as oddness, (iv) follows from the inclusion $(t+s) C \subset t C+s C$. Since $\mathcal{C}$ is closed under summation and multiplication by scalars, $\mathcal{Q}$ is a $\mathcal{C}$-valued fan.

Let

$$
L=\left\{x \in X \mid \sum_{i} x(i)=0\right\}
$$

Then $L$ is a subspace of $X$ of codimension one. Denote by $e_{j}(j \in I)$ the element of $X$ which is equal to one at $j$ and to zero at all other points of $I$. Then any nonzero $x \in L$ can be represented in the form

$$
x=\sum_{n} t_{n} e_{i_{n}}-\sum_{m} s_{m} e_{j_{m}} \quad\left(i_{n} \neq j_{m}\right)
$$

where $t_{n}>0, s_{m}>0$ and $\Sigma t_{n}=\Sigma s_{m}$. Let $q_{n m}=\left(t_{n} s_{m}\right) / k$ where $k=\Sigma t_{n}$. Then $q_{n m}>0$. Since all sets in $\mathcal{C}$ are convex, we have

$$
\mathcal{Q}(x)=\sum_{n, m} q_{n m}\left(C_{i_{n}}-C_{j_{m}}\right),
$$

hence $0 \in \mathbb{Q}(x)$ for any $x \in L$. 
According to (b), there is a selection of $Q$ defined on all of $X$ and vanishing on $L$. By Lemma 5, this implies that $\cap_{x \in L} \mathscr{Q}(x+u) \neq \varnothing$ whenever $u \notin L$. Since $e_{i}-e_{j} \in L$ for all $i, j$, it follows that

$$
\bigcap_{i \in I} C_{i}=\bigcap_{i \in I} \mathcal{Q}\left(e_{i}\right)=\bigcap_{i \in I} \mathscr{Q}\left(\left(e_{i}-e_{j}\right)+e_{j}\right) \supset \bigcap_{x \in L} \mathcal{Q}\left(x+e_{j}\right) \neq \varnothing
$$

which completes the proof.

ACKNOWLEDGEMENT. I am thankful to the referee for helpful comments.

\section{REFERENCES}

1. W. Bonnice and R. Silverman, The Hahn-Banach theorem for finite dimensional spaces, Trans. Amer. Math. Soc. 121 (1966), 210-222.

2. The Hahn-Banach extension and the least upper bound properties are equivalent, Proc. Amer. Math. Soc. 18 (1967), 843-850.

3. M. M. Day, Normed linear spaces, Springer-Verlag, Berlin and New York, 1958.

4. A. D. Ioffe, Différentielles généralisées d'applications localement lipschitziennes d'un espace de Banach dans un autre, C. R. Acad. Sci. Paris 289 (1979), 637-640.

5. __ On foundations of convex analysis, Ann. New York Acad. Sci. 337 (1980), 103-118.

6. L. V. Kantorovič, On semi-ordered linear spaces and their applications to the theory of linear operators, Dokl. Akad. Nauk SSSR 4 (1935), 11-14.

7. B. Rodriguez-Salinas and L. Bou, A Hahn-Banach theorem for arbitrary vector spaces, Boll. Un. Mat. Ital. (4) 10 (1974), 390-393.

8. H. H. Shaefer, Topological vector spaces, Macmillan, New York, 1966.

9. R. Silverman and Ti Yen, The Hahn-Banach theorem and the least upper bound property, Trans. Amer. Math. Soc. 90 (1959), 523-526.

10. T.-O. To, The equivalence of the least upper bound property and the Hahn-Banach extension property in ordered vector spaces, Proc. Amer. Math. Soc. 30 (1971), 287-296.

Profsoyuznaya 85-1-203, Moscow 117279, U.S.S.R. 